\title{
What's New in the Development of Tuberculosis Vaccines
}

\author{
Abu Salim Mustafa \\ Department of Microbiology, Faculty of Medicine, Health Sciences Center, Kuwait University, Jabriya, Kuwait
}

Tuberculosis (TB) is a major infectious disease problem of worldwide prevalence and ranks among the top 10 causes of global mortality. The most recent estimates by the World Health Organization suggest that there were 9.4 million incidence cases, and 1.7 million people died of TB in 2009 [1]. About one third of the world population is estimated to be latently infected with Mycobacterium tuberculosis, and at least $10 \%$ of these people will develop active disease in their lifetime. In spite of worldwide efforts to control TB, the global burden of the disease is increasing, particularly among the poor developing countries of Asia and Africa [1]. This is due to many reasons, including wars and immigration, poverty and malnutrition, HIV-TB co-infection and the increasing prevalence of multi-drug resistant and excessive drug-resistant TB [1]. The worldwide control of TB requires development of new drugs, cost-effective methods/reagents for specific diagnosis and new vaccines $[2,3]$. Among these possibilities, the development of new vaccines deserves priority because effective vaccines are the best weapons to fight against infectious diseases [2].

The currently available vaccine against $\mathrm{TB}$ is a live attenuated strain of pathogenic Mycobacterium bovis known as Bacillus Calmette-Guérin (BCG). Although BCG has been widely used to vaccinate against TB since 1921, it is the most controversial vaccine in current use. This is because BCG has failed to consistently protect against the major manifestation of TB in adults, i.e. pulmonary $\mathrm{TB}$ [3]. The variations in protection have ranged from nil (e.g. in India and Malawi) to $80 \%$ (e.g. in the
(C) 2012 S. Karger AG, Basel

1011-7571/12/0213-0195\$38.00/0

Fax +4161306 1234

E-Mail karger@karger.ch

www.karger.com
Accessible online at:

www.karger.com/mpp
United Kingdom) [3]. Furthermore, as BCG is a live mycobacterium [4], it is not suitable for vaccinating immunocompromised individuals, particularly HIV/AIDS patients, due to the possibility of causing disease in such individuals. Since BCG vaccination induces positivity to the commonly used tuberculin skin test for the diagnosis of TB, it becomes difficult to use this test for diagnostic or epidemiological investigations in populations vaccinated with BCG [3]. Therefore, the development of new vaccines based on $M$. tuberculosis-specific antigens is urgently needed.

The identification of $M$. tuberculosis-specific antigens has been facilitated by advances in mycobacterial genome sequencing and the comparative genomics to identify M. tuberculosis-specific genomic regions. Such studies have identified $11 \mathrm{M}$. tuberculosis-specific genomic regions known as regions of differences (RDs), which are deleted/absent in all BCG substrains currently used in different parts of the world to vaccinate against TB [5]. In silico analysis has suggested that these RDs can potentially encode 89 proteins [5]. To identify the candidate proteins suitable for vaccine development, it is essential to identify the immunodominant proteins from the RDs that can mediate protection against TB.

Protection in TB is primarily mediated by cellular immunity involving the interaction of antigen-specific $\mathrm{T}$ cells and macrophages [6]. This interaction is often indicated by antigen-induced proliferation of $\mathrm{T}$ cells and is dependent on the interplay of cytokines secreted by these cells. Although a broad spectrum of cytokines contribute 
to protection, the T-helper type 1 (Th1) cytokines, dominated by secretion of IL-2 (responsible for proliferation of antigen-reactive T cells) and IFN- $\gamma$ (responsible for activation of macrophages to kill ingested bacilli), are considered principal mediators of protective immunity against TB [6]. Therefore, Th1 cell reactivity, indicated by antigen-induced cell proliferation and secretion of IFN- $\gamma$, has been used as a marker to detect antigens involved in protective immunity and thus suitable for the identification of candidate vaccines [6].

To be able to test for the presence of proteins/peptides of RDs suitable for vaccine development, peptide pools corresponding to the predicted proteins of $M$. tuberculosis RDs have been tested for Th1 cell responses in vitro, using peripheral blood mononuclear cells from TB patients and healthy subjects, and the results showed that peptide pools of three RDs, i.e. RD1, RD7 and RD9, induced best Th1 cell responses [7]. To identify the immunodominant proteins present in these RDs, peptide pools of individual proteins of RD1, RD7 and RD9 were further tested in Th1 cell assays. The results of these experiments showed that four proteins of RD1, i.e. PE35, PPE68, ESXA and ESXB, and two proteins of RD7 (Rv2346c and Rv2947c) and RD9 (Rv3619 and Rv3620) were the best stimulators of Th1 cells in antigen-induced proliferation and/or IFN- $\gamma$ secretion assays [6]. The studies for Th1 cell epitope mapping and HLA binding showed that all of these proteins have multiple epitopes and can be presented to Th1 cells in an HLA-promiscuous manner, which are essential characteristics for a vaccine candidate to be useful in HLA-heterogeneous human populations [6].

To test the immunogenicity of the identified immunodominant proteins in animals, the genes of the above proteins were cloned into DNA vaccine vectors capable of expressing the proteins in eukaryotic cells. The recombinant DNA vaccine constructs were evaluated for induction of antigen-specific cellular immune responses in mice by using spleen cells from mice vaccinated with the recombinant DNA constructs. The results showed that antigen-specific cellular responses were observed for a given antigen only with spleen cells of mice immunized with the homologous recombinant DNA vaccine construct [8]. In addition, mice immunized with the recombinant DNA constructs showed positive Th1-type cellular responses to the specific antigen, but not to irrelevant antigens $[8,9]$. Furthermore, testing with overlapping synthetic peptides showed that Th1-type cells recognized several epitopes of proteins used for immunization $[7,8]$. These results suggest that the DNA vaccine constructs expressing the immunodominant antigens of RDs may be useful as safer vaccine candidates against TB. Interestingly, a candidate vaccine based on one of the above proteins has been shown to induce protective immunity in mice when administered along with liposomes that provide adjuvant activity [10].

In conclusion, the studies described strengthen the hypothesis that new anti-TB vaccines based on M. tuberculosis-specific antigens are a possibility. However, an effective TB vaccine development for use in humans is a long road to travel and requires preclinical and efficacy studies in humans by using appropriate adjuvants and vaccine delivery systems.

\section{Acknowledgments}

This work was supported by Kuwait University Research Administration grants MI01/10, YM08/07 and YM01/03 and General Facility grant GM01/01.

\section{References}

1 Global tuberculosis control: epidemiology, strategy, financing. WHO Report 2009 WHO/HTM/TB/2009.411.

2 Smith R: Eradication of tuberculosis by 2050 impossible without new vaccine. BMJ 2009; 338:b1291.

3 Crampin AC, Glynn JR, Fine PE: What has Karonga taught us? Tuberculosis studied over three decades. Int J Tuberc Lung Dis 2009;13:153-164.

-4 Hesseling AC, Caldwell J, Cotton MF, Eley BS, Jaspan HB, Jennings K, Marais BJ, Nuttall J, Rabie H, Roux P, Schaaf HS: BCG vaccination in South African HIV-exposed infants - risks and benefits. S Afr Med J 2009; 99:88-91.
5 Mustafa AS: Mycobacterial gene cloning and expression, comparative genomics, bioinformatics and proteomics in relation to the development of new vaccines and diagnostic reagents. Med Princ Pract 2005;14(suppl 1): $27-34$.

6 Mustafa AS: Vaccine potential of Mycobacterium tuberculosis-specific genomic regions: in vitro studies in humans. Expert Rev Vaccines 2009;8:1309-1312.

7 Al-Attiyah R, Mustafa AS: Characterization of human cellular immune responses to novel Mycobacterium tuberculosis antigens encoded by genomic regions absent in $\mathrm{Myco}$ bacterium bovis BCG. Infect Immun 2008; 76:4190-4198.
8 Hanif SNM, Al-Attiyah R, Mustafa AS: DNA vaccine constructs expressing Mycobacterium tuberculosis-specific genes induce immune responses. Scand J Immunol 2010;72: 408-415.

-9 Hanif SN, Al-Attiyah R, Mustafa AS: Cellular immune responses in mice induced by $M$. tuberculosis PE35-DNA vaccine construct. Scand I Immunol 2011;74:554-560

-10 Ansari MA, Zubair S, Mahmood A, Gupta P, Khan AA, Gupta UD, Arora A, Owais M: RD antigen based nanovaccine imparts long term protection by inducing memory response against experimental murine tuberculosis. PLoS One 2011;6:e22889. 